\title{
Ventricular tachycardia initiated by high energy cardioversion in a patient with an implantable cardioverter defibrillator
}

\author{
Masaomi Chinushi, Yoshifusa Aizawa, Kotarou Higuchi
}

First Department of Internal Medicine, Niigata University School of Medicine, 1-754 Asahimachi Niigata 951, Japan Masaomi Chinushi Yoshifusa Aizawa Kotarou Higuchi Correspondence to: Yoshifusa Aizawa. Accepted for publication 9 December 1996

\begin{abstract}
A transvenous implantable cardioverter defibrillator (ICD) was implanted into a 58 year old woman with idiopathic dilated cardiomyopathy who had drug refractory monomorphic ventricular tachycardia (VT). Antitachycardia pacing failed to terminate the VT; termination was attempted at $24 \mathrm{~J}$, which was above the defibrillation threshold. When cardioversion at $24 \mathrm{~J}$ was delivered, VT with a different morphology and slower rate was reproducibly initiated. At $3 \mathrm{~J}$, however, the original VT was successfully terminated without initiation of the slower VT. A new VT may be induced by high energy cardioversion. This may be a manifestation of the proarrhythmic potential of ICDs.
\end{abstract}

(Heart 1997;77:373-374)

Keywords: cardioversion; ventricular tachycardia; implantable cardioverter defibrillator
A

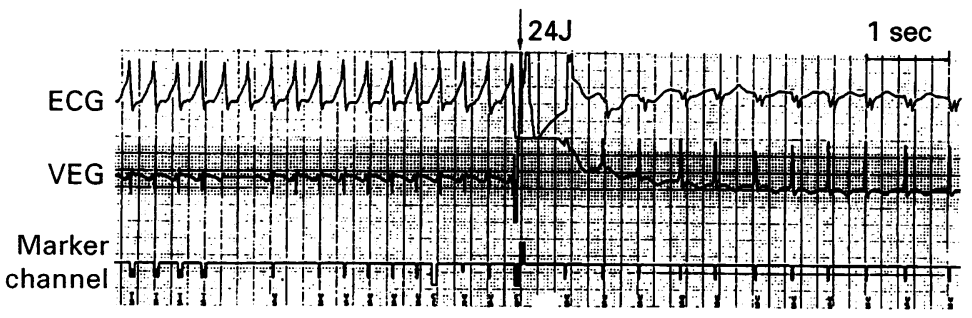

B

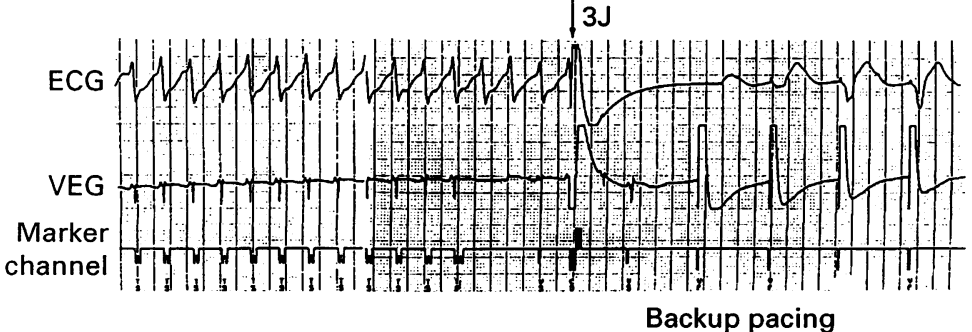

Cardioversion at $24 \mathcal{f}$ was delivered to terminate VT; the original VT was terminated but $V T$ with a new QRS morphology and a slower rate was observed immediately after the shock $(A)$. The cycle length of the new VT was longer than the tachycardia detection interval (less than $400 \mathrm{~ms}$ ), and the dysrhythmia terminated spontaneously. The initiation of new VT was avoided by cardioversion at $3 \mathcal{f}(B)$ and back-up pacings initiated after the low energy shock. ECG, lead II of surface electrocardiogram. VEG, intraventricular electrogram.
For intractable ventricular tachycardia/ ventricular fibrillation (VT/VF), transvenous implantable cardioverter defibrillators (ICD) have proved to be effective. ${ }^{2}$ However, degeneration of VT into VF may be observed in some patients especially when low energy shocks are delivered. ${ }^{3-5}$

In the present case, VT with a different QRS morphology and a slower rate was reproducibly initiated when a high energy shock $(24 \mathrm{~J})$ was delivered. Low energy cardioversion (3 J) successfully terminated the VT without initiation of a new VT.

\section{Case report}

A 58 year old woman was referred to our hospital for treatment of recurrent sustained VT. Since 1990, she has been treated with diuretics and an angiotensin converting enzyme inhibitor for idiopathic dilated cardiomyopathy. In December 1991, she complained of palpitation and fainting. Monomorphic sustained VT with four different QRS morphologies was recorded on 12-lead electrocardiograms.

On admission, chest radiography revealed mild cardiomegaly but no pulmonary congestion. A cross sectional echocardiogram showed dilatation of both ventricles and the left atrium; the wall of the left ventricle was diffusely thin and hypokinetic. Left ventriculography revealed moderate mitral regurgitation, and the ejection fraction was $36 \%$. The $12-$ lead ECG during sinus rhythm showed a complete right bundle branch block pattern with a superior axis.

After obtaining informed consent, electrophysiological study was performed using the standard technique. Four monomorphic VTs (two with left bundle branch block pattern and two with right bundle branch block pattern) were reproducibly induced by programmed stimulation. The site of each VT origin was mapped on either side of the interventricular septum (three sites) and in the free wall of the left ventricle (one site). During electrophysiological study, criteria for transient entrainment were confirmed by rapid ventricular pacing ${ }^{6}$; however, pacing at shorter cycle lengths was unable to terminate, and usually accelerated, the VT. External cardioversion had to be used to restore sinus rhythm. 
Antiarrhythmic drugs including DL-sotalol failed to prevent the induction or recurrence of VT. Radiofrequency catheter ablation was effective in eliminating only one VT morphology. The patient was considered as a candidate for ICD treatment.

In September 1995, a transvenous ICD system (Medtronic Jewel-Plus, 7220C, Active Can, Minneapolis, Minnesota, USA) was implanted. During the operation, a shock of $24 \mathrm{~J}$ was twice confirmed capable of terminating VF. A shock of $24 \mathrm{~J}$ was set as the first treatment for VF and VT.

Two days after the operation, VT recurred and cardioversion at $24 \mathrm{~J}$ was appropriately delivered between the body of ICD generator and the common lead in the right ventricle. However, VT with a non-clinical QRS morphology and longer cycle length was initiated immediately by the shock (fig). The VT showed a gradual prolongation of the $R-R$ interval and terminated spontaneously within 10 seconds. The same phenomenon was reproduced after administration of antiarrhythmic drugs (aprindine and bepridil hydrochloride). The energy of the first shock was then set at $3 \mathrm{~J}$ which successfully terminated VT without initiation of the non-clinical VT (fig).

\section{Discussion}

In the present case, the mechanism of the nonischaemic VT was shown, by confirming the criteria for transient entrainment, to be reentry with an excitable gap. ${ }^{67}$ However, VT could not be terminated with rapid pacing ${ }^{8}$ and termination of VT/VF was attempted by the delivery of shock at $24 \mathrm{~J}$.

Immediately after the shock, VT with different morphology was reproducibly induced. The non-clinical VT was characterised by a gradual slowing and spontaneous termination; automaticity seems to be the likely mecha- nism. VT might be induced by shock delivery $^{3-5}$ but characteristics of the shock induced VT has not been fully described. Interestingly, the induction of VT was prevented by a shock of $3 \mathrm{~J}$ which successfully terminated the original VT.

Shocking with high energy may enhance automaticity in some diseased myocardium, ${ }^{910}$ and may have been the mechanism of the phenomenon seen in the present case. Mechanical stimulation by an implanted lead could be excluded because the QRS morphology of the non-clinical VT was different from that of the paced one at the site (fig).

1 Powell AC, Fuchs P, Finkelstein DM, Garan H, Cannom DS, McGovern VA, et al. Influence of the automatic implantable cardioverter-defibrillator on the long-term prognosis of survivors of out-of-hospital cardiac arrest. Circulation 1993;88:1083-92.

2 Winkle RA, Mead RH, Ruder MA, Gaudiani VA, Smith NA, Buch WS, et al. Long-term outcome with the automatic implantable cardioverter-defibrillator. $\mathcal{f} \mathrm{Am}$ Coll matic implantable cardio

3 Lauer MR, Young C, Liem LB, Ottoboni L, Peterson J, Goold P, et al. Ventricular fibrillation induced by lowenergy shocks from programmable implantable carenergy shocks from programmable implantable car-
dioverter-defibrillators in patients with coronary artery dioverter-defibrillators in patients with
disease. Am $\mathcal{F}$ Cardiol 1994;73:559-63.

4 Pinski SL, Fahy GJ. The proarrhythmic potential of implantable cardioverter-defibrillators. Circulation 1995; 92:1651-64.

5 Cohen TJ, Chien WW, Lurie KG, Lee MA, Lesh MD, Scheinman MM, et al. Implantable cardioverter defibrillator proarrhythmia: case report and review of the literature. PACE 1991;14:1326-429.

6 Waldo AL, Henthorn RW, Plumb VJ, MacLean WAH Demonstration of the mechanism of transien entrainment and interruption of ventricular tachycardia with rapid atrial pacing. I Am Coll Cardiol 1984;3: 422-30.

7 Aizawa Y, Naitoh N, Kusano Y, Uchiyama H, Washizuka $T$, Shibata A. Frequency of presumed reentry with an excitable gap in sustained ventricular tachycardia unassociated with coronary artery disease. Am f Cardiol 1993; 72:916-21.

8 Aizawa Y, Niwano S, Chinushi M, Tamura M, Kusano Y, Kitazawa $\mathrm{H}$, et al. Incidence and the mechanism of interruption of ventricular tachycardia with rapid ventricular pacing. Circulation 1992;82:589-95.

9 Levine JH, Spear JF, Weisman HF, Kadish AH, Prood C, Siu CO, et al. The cellular electrophysiologic changes induced by high-energy electrical ablation in canine myocardium. Circulation 1986;73:818-29.

10 Kodama I, Shibata N, Sakuma I, Mitsui K, Iida M, Suzuki $\mathrm{R}$, et al. After effects of high-intensity DC stimulation on the electromechanical performance of ventricular muscle. Am $\mathcal{F}$ Physiol 1994;267(Heart Circ Physiol 36): 